\title{
Quantifying Intracranial Internal Carotid Artery Stenosis on MR Angiography
}

\author{
(D) H. Baradaran, (DP. Patel, (D) G. Gialdini, (D). Al-Dasuqi, (D) A. Giambrone, DH. Kamel, and (D) A. Gupta
}

\begin{abstract}
BACKGROUND AND PURPOSE: Intracranial atherosclerosis is a common cause of ischemic stroke. Intracranial stenosis is most commonly quantified by the Warfarin-Aspirin Symptomatic Intracranial Disease method, which involves calculating a ratio of luminal diameter measurements on conventional angiography. Our purpose was to determine whether a single linear measurement of the narrowest caliber of the intracranial ICA on MRA can accurately predict Warfarin-Aspirin Symptomatic Intracranial Disease stenosis measurements.
\end{abstract}

MATERIALS AND METHODS: We identified patients from a prospective stroke registry who had undergone head MRAs to quantitatively evaluate the degree of Warfarin-Aspirin Symptomatic Intracranial Disease-derived stenosis in each intracranial ICA. We also made a single linear millimeter measurement at the site of maximal narrowing of the ICA. We calculated a correlation coefficient between the lumen diameter in millimeters and percentage Warfarin-Aspirin Symptomatic Intracranial Disease stenosis. We performed receiver operating characteristic analysis to determine optimal luminal diameter cutoff values.

RESULTS: In 386 unique intracranial ICAs, we found a strong linear relationship between single lumen measurements and Warfarin-Aspirin Symptomatic Intracranial Disease-style stenosis measurements $(R=-0.84, P<.0001)$. We found that ICA lumen diameters of $\leq 2.1$ and $\leq 1.3 \mathrm{~mm}$ were optimal cutoffs for identifying patients with $\geq 50 \%$ stenosis and $\geq 70 \%$ stenosis, respectively (area under the curve $=0.96$ and 0.99 , respectively).

CONCLUSIONS: There is a strong linear relationship between the narrowest lumen diameter of the intracranial ICA and percentage stenosis. Our results suggest that a single lumen diameter measurement on MRA allows accurate estimation of Warfarin-Aspirin Symptomatic Intracranial Disease stenosis, which may affect risk stratification and treatment decisions.

ABBREVIATIONS: CAESAR = Cornell AcutE Stroke Academic Registry; WASID = Warfarin-Aspirin Symptomatic Intracranial Disease

ntracranial atherosclerotic disease is a major risk factor for ischemic stroke. As one of the most common causes of stroke worldwide, ${ }^{1}$ intracranial atherosclerosis is generally assessed by measuring the degree of luminal stenosis on angiography. ${ }^{2,3}$ Ac-

Received July 12, 2016; accepted after revision December 14.

From the Department of Radiology (H.B., P.P., K.A.-D., A. Gupta), Clinical and Trans lational Neuroscience Unit (H.B., G.G., H.K., A. Gupta), Feil Family Brain and Mind Research Institute, Department of Healthcare Policy and Research (A. Giambrone), and Department of Neurology (H.K.), Weill Cornell Medicine, New York, New York.

Dr Ajay Gupta is supported by National Institutes of Health/National Center for Advancing Translational Sciences grant No. KL2TR000458; and Dr Hooman Kamel is supported by National Institutes of Health/National Institute of Neurological Disorders and Stroke grant No. K23NS082367.

Please address correspondence to Ajay Gupta, MD, Department of Radiology, Weill Cornell Medicine, 525 East 68th St, Box 141 Starr 8A, New York, NY 10065; e-mail: ajg9004@med.cornell.edu

- Indicates open access to non-subscribers at www.ajnr.org

EIndicates article with supplemental on-line table.

Indicates article with supplemental on-line photo.

http://dx.doi.org/10.3174/ajnr.A5113 curate assessment of the degree of luminal stenosis of the cerebral vasculature is important to assign stroke etiology because most stroke classification schemes require a luminal stenosis of $\geq 50 \%$ for a stroke to be attributed to large-vessel atherosclerosis. ${ }^{4}$ In addition, given trial data showing that aggressive medical therapy is an effective approach to reduce recurrent stroke risk in patients with $\geq 70 \%$ stenosis of a major intracranial artery, ${ }^{5}$ reliable and accurate stenosis measurements are critical for appropriate patient selection for preventative therapy. ${ }^{4}$ Similarly, studies have shown that patients with $\geq 70 \%$ stenosis are at an elevated risk of stroke recurrence compared with those with 50\%-69\% stenosis. ${ }^{3}$

The most commonly used method for calculating intracranial stenosis severity is the Warfarin-Aspirin Symptomatic Intracranial Disease (WASID) method, which has been the basis of several randomized controlled trials for the treatment of major intracranial stenosis. ${ }^{5-7}$ The measurement is performed by calculating a ratio of luminal diameters obtained from angiographic images. Although the WASID technique has high reproducibility with experienced readers and meticulous technique, the practical imple- 


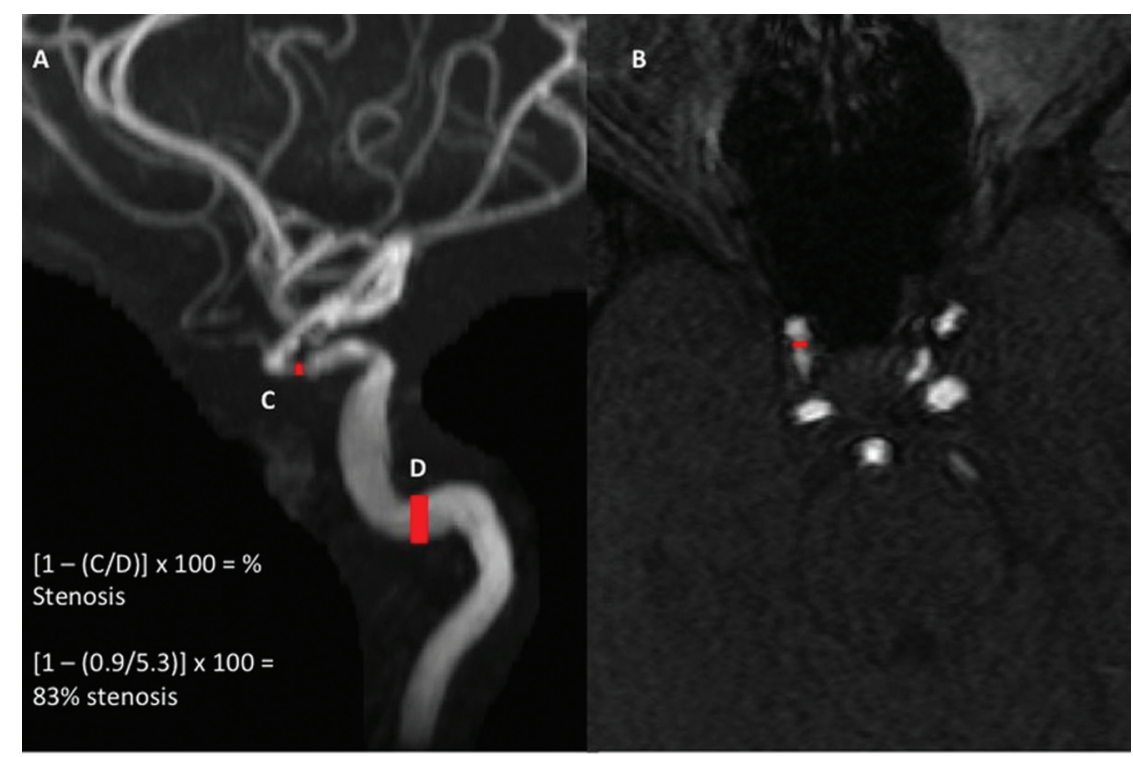

FIG 1. MRA MIP $(A)$ and axial source $(B)$ images demonstrating high-grade stenosis of the right intracranial ICA. For an accurate measurement of the degree of Warfarin-Aspirin Symptomatic Intracranial Disease stenosis, we made a linear measurement of the most stenotic portion of the ICA on either the MIP or axial source image, in this case $0.9 \mathrm{~mm}(C)$. A second "normal" measurement was made at the widest, nontortuous, normal portion of the petrous ICA that had margins parallel with the site of stenosis, in this case $5.3 \mathrm{~mm}(D)$. If the petrous ICA was also diseased, the most distal, parallel part of the extracranial ICA was measured. The ratio of these 2 measurements was then used to calculate the WASID stenosis, in this case $[1-(0.9 / 5.30)] \times 100=83 \%$ stenosis. Alternatively, we could use the single linear measurement at the site of greatest stenosis, $0.9 \mathrm{~mm}$ (C), which correlates with a stenosis of $>70 \%$. vascular risk factors (including atrial fibrillation, tobacco use, diabetes mellitus, hypertension, dyslipidemia, peripheral vascular disease, and cardiac valvular disease) were prospectively collected by trained hospital personnel. Retrospectively, 2 neurologists used available medical records to independently identify stroke etiology by using the Trial of Org 10172 in Acute Stroke Treatment classification scheme, ${ }^{4}$ with a third neurologist to independently resolve disagreements.

All included patients underwent noncontrast MRA examinations on either a $1.5 \mathrm{~T}$ or 3T Signa (GE Healthcare, Milwaukee, Wisconsin) scanner. 3DTOF acquisitions of the head were performed with an FOV of $20 \mathrm{~cm}, 1.4-\mathrm{mm}$ section thickness, $\mathrm{TR}=25, \mathrm{TE}=3$, and matrices of $320 \times 192$ and $3210 \times 224$ on $1.5 \mathrm{~T}$ and $3 \mathrm{~T}$ scanners, respectively. Maximum intensity projections of each intracranial ICA were created.

We measured the degree of ICA narrowing by using the WASID method $^{6}$ and single luminal measurements. mentation of WASID measurements can be cumbersome and prone to error, especially in the ICA where multiple vascular segments and an anatomically tortuous course can complicate measurement. For these reasons, strictly defined WASID stenosis measurements are not routinely performed in day-to-day practice, especially with noninvasive vascular imaging modalities. Thus, having a rapid and accurate method of estimating luminal stenosis measurements by using only single-diameter measurements obtained on cross-sectional images would be beneficial, similar to prior work done in the extracranial carotid artery. ${ }^{8}$ The purpose of our study was therefore to test the hypothesis that a single luminal measurement on MRA images of the ICA can accurately approximate the WASID-determined intracranial ICA stenosis in a cohort of patients with acute ischemic stroke.

\section{MATERIALS AND METHODS}

Patients admitted to New York Presbyterian Hospital/Weill Cornell Medical Center with acute ischemic stroke in 2013 were included in the prospectively maintained Cornell AcutE Stroke Academic Registry (CAESAR). We retrospectively included all patients with MRA of the head because we were interested in characterizing the degree of intracranial arterial stenosis. We excluded any patients with complete occlusion of their intracranial ICAs and any patients with images that were too motion-degraded for accurate interpretation and measurement. The Weill Cornell Medicine institutional review board approved this data collection and waived the need for informed consent, given the retrospective nature of the analysis and minimal risk to subjects.

As part of the CAESAR registry, data on patient demographics, National Institutes of Health Stroke Scale score on admission, and

\section{WASID Measurement Technique}

To calculate WASID stenosis, we obtained 2 measurements for each intracranial ICA: 1) a linear measurement at the site of the most severe stenosis on either the MIP or axial source images; and 2) a linear measurement at the widest, nontortuous, normal portion of the petrous ICA parallel to the site of stenosis. Using these measurements, we calculated the degree of WASID stenosis by using the following equation: Percentage Stenosis $=\left[\left(1-\left[\mathrm{D}_{\text {stenosis }} / \mathrm{D}_{\text {normal }}\right]\right)\right] \times$ 100 , where $D_{\text {stenosis }}$ is the diameter of the artery at the site of most severe degree of stenosis and $\mathrm{D}_{\text {normal }}$ is the diameter of the proximal artery at its widest, nontortuous, normal segment. ${ }^{6}$

\section{Single Luminal Measurement Technique}

For the single luminal measurement calculations, a single luminal measurement was made at the site of the most severe stenosis within the intracranial ICA.

The stenosis measurements for both techniques were obtained in either the cavernous or supraclinoid segments of the ICA. In cases of no measurable stenosis, linear measurements were made in the normal-caliber cavernous segment of the intracranial ICA. Stenosis was evaluated and measurements were made by using a combination of the MIP and axial source MRA images. Linear measurements were obtained on the MIP projection or axial source image showing the greatest degree of stenosis (Fig 1 and On-line Fig 1). Measurements were made from outer lumen to outer lumen in all arteries. A second radiologist independently calculated WASID stenosis measurements of the first 50 consecutive intracranial ICAs ordered by admission date to evaluate interobserver reproducibility. 


\section{Statistical Analysis}

Categoric data are presented as number (percentage); and continuous data, as mean $\pm \mathrm{SD}$. Pearson correlation coefficients were used to determine the strength of the linear relationship between millimeter stenosis and percentage WASID stenosis followed by linear regression to determine $95 \%$ confidence intervals for the percentage stenosis predicted values at each millimeter stenosis.

\section{Table 1: Patient demographics}

\begin{tabular}{lc}
\hline \multicolumn{1}{c}{ Variable } & No. (\%) \\
\hline Age (yr) (mean) & $71.9 \pm 14.0$ \\
Female & $91(47.2)$ \\
Race & \\
$\quad$ White & $173(89.6)$ \\
$\quad$ Black & $11(5.7)$ \\
Other & $9(4.7)$ \\
Atrial fibrillation & $27(14.0)$ \\
Coronary artery disease & $37(19.2)$ \\
Carotid artery disease & $7(3.6)$ \\
Diabetes & $55(28.5)$ \\
Hypertension & $132(68.4)$ \\
Dyslipidemia & $103(53.4)$ \\
NIHSS quartile & \\
1 & $54(28.0)$ \\
2 & $48(24.9)$ \\
3 & $22(11.4)$ \\
4 & $69(35.8)$ \\
Prior stroke & $63(32.6)$ \\
Peripheral vascular disease & $13(6.7)$ \\
Active tobacco use & $18(9.3)$ \\
Stroke subtype & \\
Cardioembolic & $62(32.1)$ \\
Cryptogenic & $77(39.9)$ \\
Large-artery atherosclerosis & $25(13.0)$ \\
Small-vessel occlusion & $20(10.4)$ \\
Other & $9(4.7)$ \\
IV tPA administered & $16(8.3)$ \\
Valvular disease & $4(2.1)$ \\
\hline
\end{tabular}

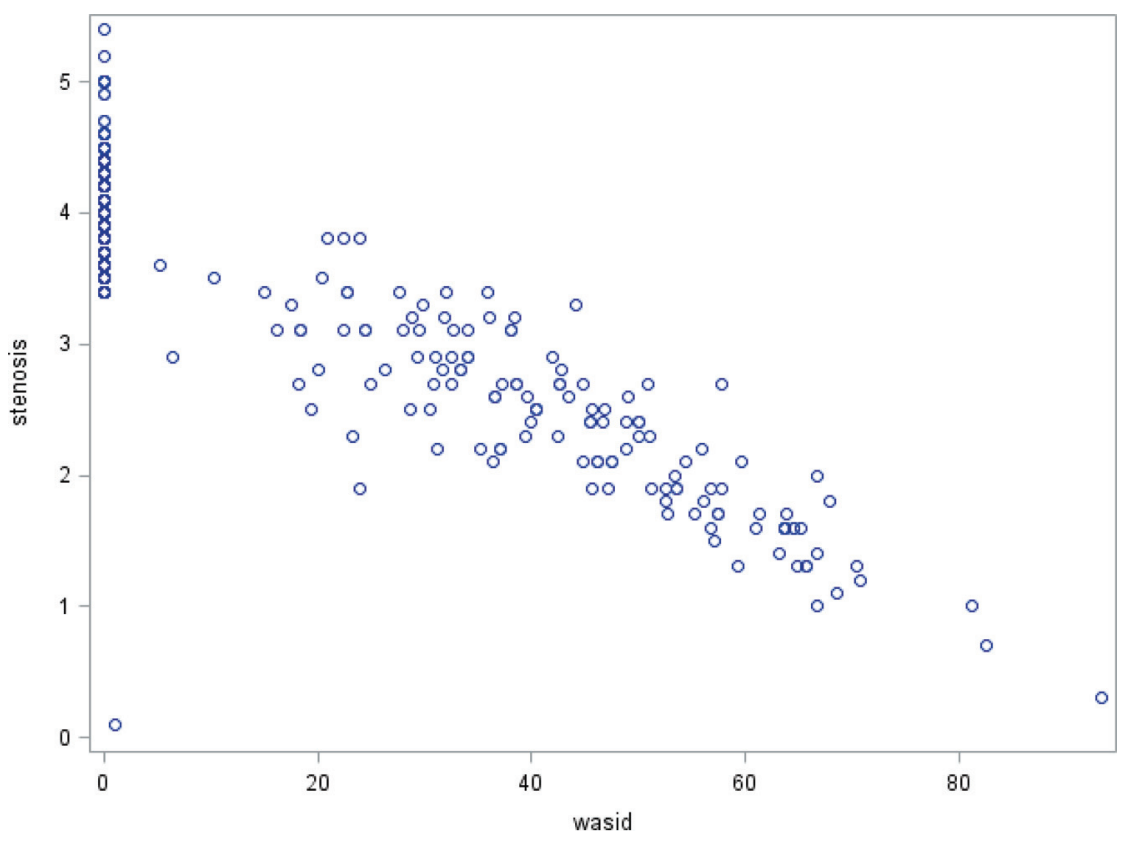

FIG 2. Correlation scatterplot demonstrating a linear relationship between millimeter stenosis measurements and percentage WASID stenosis for all included patients with $R=-0.84(P<$ $.0001)$.
Pearson correlation coefficients were also performed on subgroups stratified by magnet field strength (1.5T versus $3 \mathrm{~T}$ ). WASID and millimeter stenosis measurements were each divided into 3 groups ( $<50 \%$ stenosis, $50 \%-69 \%$ stenosis, and $\geq 70 \%$ stenosis) to determine the number of arteries falling into each category. Sensitivity, specificity, positive predictive value, and negative predictive value were calculated for the millimeter stenosis measurement in discriminating between $50 \%$ and $70 \%$ stenosis, respectively. The optimal threshold for both $50 \%$ and $70 \%$ stenosis was determined by using the Youden index and receiver operating characteristic curves. All $P$ values were 2 -sided and were evaluated at the $.05 \alpha$ level. All analyses were performed by using SAS, Version 9.3 (SAS Institute, Cary, North Carolina).

\section{RESULTS}

Of the 335 patients included in the 2013 CAESAR stroke registry, 134 patients were excluded because they did not have an MRA examination of their head; 7 patients, because they had complete occlusion of their intracranial ICA; and 1 patient, because his MRA was too motion-degraded for accurate measurement. Of the 193 patients (386 arteries) who were ultimately included, the mean age was 71.9 years (Table 1 ).

Measures of single lumen diameter taken at the narrowest point of the intracranial ICA and the percentage WASID stenosis showed a linear relationship with excellent correlation $(R=$ $-0.84, P<.0001$ ) (Fig 2). A subset analysis demonstrated similar correlation coefficients across $1.5 \mathrm{~T}$ and $3 \mathrm{~T}$ machines with $R=$ $-0.85(P<.001)$ and $-0.82(P<.001)$, respectively. We also calculated the WASID percentage stenosis corresponding to the narrowest ICA lumen diameter in increments of $0.1 \mathrm{~mm}$ (Table 2).

Using receiver operating curve analysis on the 139 arteries $(36 \%)$ with detectable WASID stenosis (Table 3), we determined that a millimeter measurement of 2.1 mm predicts $>50 \%$ stenosis (area under the curve $=0.96$, sensitivity $=89.9$, specificity $=86.0$ ) and a millimeter measurement of $1.3 \mathrm{~mm}$ predicts $>70 \%$ stenosis (area under the curve $=0.99$, sensitivity $=95.5$, specificity $=100$ ) (Fig 3 and On-line Table).

Of the 247 arteries without measurable WASID stenosis, the mean millimeter measurement was $3.97 \pm 0.3 \mathrm{~mm}$ (range, 2.6-5.4 mm) and a median of $3.9 \mathrm{~mm}$ (interquartile range, 3.8-4.2).

Measures of interobserver reproducibility showed a $\kappa$ coefficient of 0.85 (range, 0.72-0.98) for millimeter stenosis measurements.

\section{DISCUSSION}

In our analysis, we found a strong linear relationship with excellent correlation between millimeter measurement of the narrowest point of the ICA and percentage WASID stenosis. Additionally, we were able to determine optimal millime- 
Table 2: WASID percentage stenosis estimates from millimeter measurements of the most stenotic portion of the intracranial ICA

\begin{tabular}{lc}
\hline Stenosis $(\mathrm{mm})$ & \% WASID Stenosis $(\mathbf{9 5 \%} \mathrm{Cl})$ \\
\hline $3.0-3.8$ & $14-30$ \\
$2.2-2.9$ & $31-48$ \\
$1.3-2.2$ & $50-65$ \\
$<1.3$ & $>65$ \\
\hline
\end{tabular}

Table 3: Breakdown of the millimeter measurements of the 139 arteries with detectable WASID stenosis

\begin{tabular}{lcccc}
\hline $\begin{array}{c}\text { Stenosis } \\
\text { Measurement }\end{array}$ & $<50 \%$ & $\mathbf{5 0 \% - 6 9 \%}$ & $\mathbf{7 0 \% +}$ & Total \\
\hline$>2.1 \mathrm{~mm}$ & 75 & 6 & 0 & 81 \\
$1.4-2.1 \mathrm{~mm}$ & 14 & 32 & 0 & 46 \\
$\leq 1.3 \mathrm{~mm}$ & 0 & 6 & 6 & 12 \\
Total & 89 & 44 & 6 & 139 \\
\hline
\end{tabular}
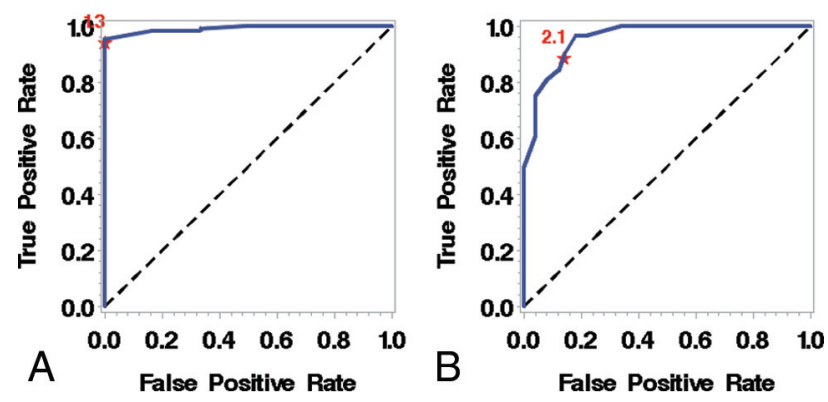

FIG 3. $A$, Receiver operating characteristic curve for predicting $>70 \%$ stenosis demonstrates an optimal cutoff of $1.3 \mathrm{~mm}$. B, Receiver operating characteristic curve for predicting $>50 \%$ stenosis demonstrates that a measurement of $2.1 \mathrm{~mm}$ is an ideal cutoff.

ter cutoffs of 2.1 and $1.3 \mathrm{~mm}$ to identify those with $\geq 50 \%$ and $\geq 70 \%$ stenosis respectively by WASID methods, which demonstrated excellent accuracy. These findings are important because they suggest that accurate luminal stenosis measurements for the intracranial ICA can be obtained without calculating ratios, which are cumbersome and may be prone to error. We believe that a single luminal diameter measurement offers a simple and rapid approach to ICA stenosis measurement that can be readily integrated into clinical practice. Accurate quantification of intracranial ICA stenosis is important because it allows clinicians to determine whether patients exceed specific stenosis thresholds above which intensive medical therapies are warranted, according to existing evidence-based guidelines.

We used MR angiographic studies to evaluate the degree of stenosis. Although the original WASID measurements were performed on conventional angiography, the high radiation exposure, need to administer iodinated contrast, stroke risk, and risks of arterial puncture have reduced the role of conventional angiography in screening for atherosclerosis. ${ }^{9}$ Conversely, MRA is an attractive technique for screening intracranial stenosis for several reasons: First, MR angiographic studies are obtained relatively quickly and are often performed concurrently with brain MR imaging in the evaluation of patients with potential stroke. At many institutions, including our own, cross-sectional imaging is almost exclusively used for the evaluation of intracranial stenosis and to inform treatment decisions, including whether to initiate more intensive medical therapy for stroke prevention. Second, using
MIPs from MRA studies simulates the appearance of conventional angiography, thereby allowing measurements to be obtained in the same locations and projections, similar to those for the original WASID measurements. Third, recent studies have shown that MRA performs well, with sensitivities and specificities ranging from $80 \%$ to $100 \%$ and $89 \%$ to $95 \%$, respectively, compared with conventional angiography when assessing intracranial stenosis. ${ }^{10-13}$ Furthermore, MRA is not subject to the challenge of separating a high-density contrast-enhanced lumen from adjacent calcification and bone inherent in the evaluation of CTA of the head.

Our study had some limitations. First, time-of-flight MR angiography is sensitive to flow disturbances caused by stenosis ${ }^{14}$ and is known to overestimate the degree of stenosis compared with CT and conventional angiography. ${ }^{13}$ By evaluating both the MIP and axial source images to measure the degree of stenosis, according to established methods ${ }^{15}$ shown to be highly accurate, we attempted to minimize the degree of overestimation of stenosis. ${ }^{10-13}$ Second, obtaining measurements on MR angiograms can be subject to error, given the lack of precise spatial resolution. We used a uniform method for making all WASID measurements, including measuring from outer lumen to outer lumen. Additionally, we had excellent interobserver reliability between our 2 readers. Last, we had a relatively small number of ICAs demonstrating high-grade intracranial stenosis, with an overall prevalence of $12.9 \%$ for a stenosis of $\geq 50 \%$ and $1.6 \%$ for a stenosis of $\geq 70 \%$. The relatively low prevalence of high-grade stenosis likely contributed to the positive predictive value of $50 \%$ for the cutoff for $>70 \%$ stenosis.

We believe that by focusing on all ICAs in our cohort regardless of stenosis severity, our results are more generalizable, especially given that our data are consistent with the prevalence data of high-grade intracranial ICA stenosis derived from populationbased studies. ${ }^{16}$ Because we did not limit our study sample exclusively to patients with high-grade stenosis, the precision of our cutoff values to estimate exact degrees of high-grade WASID stenosis values is somewhat limited. We believe that our cutoff values are most useful as a practical and rapid approach to screen for potentially clinically relevant intracranial atherosclerotic stenosis, whose identification may warrant more detailed evaluation and possible treatment. Validating these cutoff values further in a separate prospective cohort would also be valuable.

\section{CONCLUSIONS}

We found a strong linear relationship between a simple measurement of the narrowest point of the intracranial ICA and the percentage WASID stenosis. Our results allow a direct estimation of WASID stenosis via a single-diameter measurement on MRA. We found an optimal cutoff measurement of $2.1 \mathrm{~mm}$ for identifying patients with $\geq 50 \%$ stenosis and $1.3 \mathrm{~mm}$ for identifying patients with $\geq 70 \%$ stenosis and that there is a strong linear relationship between the narrowest lumen diameter of the intracranial ICA and WASID-derived percentage stenosis, allowing a single linear measurement to provide accurate WASID stenosis estimates, which are useful in guiding treatment decisions. 
Disclosures: Ajay Gupta—RELATED: Grant: National Institutes of Health, Comments: KL2 TR000458 supporting Dr Gupta, administered through Weill Cornell Clinical and

Translational Science Center. * *Money paid to the institution.

\section{REFERENCES}

1. Holmstedt CA, Turan TN, Chimowitz MI. Atherosclerotic intracranial arterial stenosis: risk factors, diagnosis, and treatment. Lancet Neurol 2013;12:1106-14 CrossRef Medline

2. Gorelick PB, Wong KS, Bae HJ, et al. Large artery intracranial occlusive disease a large worldwide burden but a relatively neglected frontier. Stroke 2008;39:2396-99 CrossRef Medline

3. Kasner SE, Chimowitz MI, Lynn MJ, et al; Warfarin Aspirin Symptomatic Intracranial Disease Trial Investigators. Predictors of ischemic stroke in the territory of a symptomatic intracranial arterial stenosis. Circulation 2006;113:555-63 CrossRef Medline

4. Adams HP Jr, Bendixen BH, Kappelle LJ, et al. Classification of subtype of acute ischemic stroke: definitions for use in a multicenter clinical trial-TOAST. Trial of Org 10172 in Acute Stroke Treatment. Stroke 1993;24:35-41 CrossRef Medline

5. Chimowitz MI, Lynn MJ, Derdeyn CP, et al; SAMMPRIS Trial Investigators. Stenting versus aggressive medical therapy for intracranial arterial stenosis. N Engl J Med 2011;365:993-1003 CrossRef Medline

6. Samuels OB, Joseph GJ, Lynn MJ, et al. A standardized method for measuring intracranial arterial stenosis. AJNR Am J Neuroradiol 2000;21:643-46 Medline

7. Chimowitz MI, Lynn MJ, Howlett-Smith H, et al; Warfarin-Aspirin Symptomatic Intracranial Disease Trial Investigators. Comparison of warfarin and aspirin for symptomatic intracranial arterial stenosis. N Engl J Med 2005;352:1305-16 CrossRef Medline

8. Bartlett ES, Walters TD, Symons SP, et al. Quantification of carotid stenosis on CT angiography. AJNR Am J Neuroradiol 2006;27:13-19 Medline
9. Willinsky RA, Taylor SM, terBrugge K, et al. Neurologic complications of cerebral angiography: prospective analysis of 2,899 procedures and review of the literature. Radiology 2003;227:522-28 CrossRef Medline

10. Oelerich M, Lentschig GM, Zunker P, et al. Intracranial vascular stenosis and occlusion: comparison of 3D time-of-flight and 3D phase-contrast MR angiography. Neuroradiology 1998;40:567-73 CrossRef Medline

11. Choi CG, Lee DH, Lee JH, et al. Detection of intracranial atherosclerotic steno-occlusive disease with $3 \mathrm{D}$ time-of-flight magnetic resonance angiography with sensitivity encoding at 3T. AJNR Am J Neuroradiol 2007;28:439-46 Medline

12. Hirai T, Korogi Y, Ono K, et al. Prospective evaluation of suspected stenoocclusive disease of the intracranial artery: combined MR angiography and CT angiography compared with digital subtraction angiography. AJNR Am J Neuroradiol 2002;23:93-101 Medline

13. De Marco J, Nesbit G, Wesbey G, et al. Prospective evaluation of extracranial carotid stenosis: MR angiography with maximum-intensity projections and multiplanar reformation compared with conventional angiography. AJR Am J Roentgenol 1994;163:1205-12 CrossRef Medline

14. Bash S, Villablanca JP, Jahan R, et al. Intracranial vascular stenosis and occlusive disease: evaluation with CT angiography, MR angiography, and digital subtraction angiography. AJNR Am J Neuroradiol 2005;26:1012-21 Medline

15. Korogi $\mathrm{Y}$, Takahashi M, Nakagawa $\mathrm{T}$, et al. Intracranial vascular stenosis and occlusion: MR angiographic findings. AJNR Am J Neuroradiol 1997;18:135-43 Medline

16. Suri MF, Qiao Y, Ma X, et al. Prevalence of intracranial atherosclerotic stenosis using high-resolution magnetic resonance angiography in the general population: the Atherosclerosis Risk in Communities Study. Stroke 2016;47:1187-93 CrossRef Medline 